\title{
Metal Oxide Nanoparticle Mediated Enhanced Raman Scattering and Its Use in Direct Monitoring of Interfacial Chemical Reactions
}

\author{
$\mathrm{Li} \mathrm{Li}^{\dagger}{ }^{\dagger}$ Tanya Hutter, ${ }^{\ddagger}$ Alexander S. Finnemore, ${ }^{\dagger}$ Fu Min Huang, ${ }^{\dagger}$ Jeremy J. Baumberg, ${ }^{\dagger}$ \\ Stephen R. Elliott, ${ }^{\ddagger}$ Ullrich Steiner, ${ }^{\dagger}$ and Sumeet Mahajan* ${ }^{\dagger}$ \\ ${ }^{\dagger}$ Department of Physics, Cavendish Laboratory, University of Cambridge, Cambridge CB3 OHE, U.K. \\ ${ }^{\ddagger}$ Department of Chemistry, University of Cambridge, Lensfield Road, Cambridge CB2 1EW, U.K.
}

\section{Supporting Information}

\begin{abstract}
Metal oxide nanoparticles (MONPs) have widespread usage across many disciplines, but monitoring molecular processes at their surfaces in situ has not been possible. Here we demonstrate that MONPs give highly enhanced $\left(\times 10^{4}\right)$ Raman scattering signals from molecules at the interface permitting direct monitoring of their reactions, when placed on top of flat metallic surfaces. Experiments with different metal oxide materials and

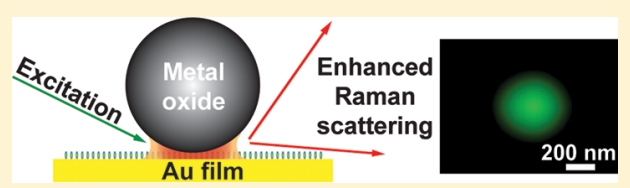
molecules indicate that the enhancement is generic and operates at the single nanoparticle level. Simulations confirm that the amplification is principally electromagnetic and is a result of optical modulation of the underlying plasmonic metallic surface by MONPs, which act as scattering antennae and couple light into the confined region sandwiched by the underlying surface. Because of additional functionalities of metal oxides as magnetic, photoelectrochemical and catalytic materials, enhanced Raman scattering mediated by MONPs opens up significant opportunities in fundamental science, allowing direct tracking and understanding of application-specific transformations at such interfaces. We show a first example by monitoring the MONPassisted photocatalytic decomposition reaction of an organic dye by individual nanoparticles.
\end{abstract}

KEYWORDS: Metal oxide, plasmons, surface-enhanced Raman scattering, photocatalysis, interface

$\mathrm{T}$ ransition-metal oxides, due to the strong correlations of their d electrons, give rise to a wide variety of phenomena such as magnetism, ionic conduction, metal-insulator transitions, multiferroicity, and superconductivity. ${ }^{1}$ As a result, they have an extensive range of applications that include fuel cells, batteries, catalysts, sensors, and microelectronics. ${ }^{1}$ Despite the resulting importance of molecular binding and surface reactivity, their utilization in plasmonic applications has been prevented by the tuning of their localized surface plasmon resonance (LSPR) into the infrared. ${ }^{2-6}$ Surface-enhanced Raman scattering (SERS) is a popular plasmonic application utilizing ultraviolet (UV), visible (VIS), or near-infrared (NIR) excitation, which overcomes the extremely small scattering cross section $\left(\sim 10^{-30} \mathrm{~cm}^{2}\right.$ per molecule $)$ in conventional Raman scattering ${ }^{7}$ to yield a technique that offers noninvasive and nondestructive fingerprint characterization ${ }^{8}$ with extensive applications in chemical and biological sensing. The amplification in SERS stems primarily from the electromagnetic (EM) enhancement (up to $\left.10^{14}\right)^{9}$ obtained by excitation of SPR. ${ }^{10}$ This is accompanied by typically smaller and system-dependent chemical enhancement as a result of formation of chargetransfer complexes between adsorbate and the surface. ${ }^{11}$ Therefore, for efficient and sensitive SERS detection of molecules, nanoscale structures fabricated entirely with coinage metals (especially Ag and $\mathrm{Au}$ ) have been the materials of choice since their SPR is easily excited in the vis or NIR regions. On the other hand, use of metal oxide nanoscale materials for enhanced Raman scattering has remained confined to few charge-transfer complexes, ${ }^{12-14}$ limiting its widespread application to interfaces.

In this work, we demonstrate that Raman scattering can be greatly amplified just by placing metal oxide nanoparticles (MONPs) on flat metallic surfaces. In order to differentiate this approach from the various metallic NP (or NP array) based SERS strategies, ${ }^{15,16}$ we christen it metal oxide nanoparticleenhanced Raman scattering (MONERS). Although its construction is analogous, it is unlike systems where the enhancements are due to coupling between the LSPR of the metallic NP and the propagating surface plasmon polaritons of the underlying metallic substrate ${ }^{17,18}$ or the modification of LSPR modes of the plasmonic NP by a dielectric substrate. ${ }^{19,20}$ In this case the MONPs are nonplasmonic and do not have a LSPR in the wavelength range studied. Further, our method is generic and applicable to a variety of MONPs fabricated from materials such as $\mathrm{Fe}_{3} \mathrm{O}_{4}, \mathrm{TiO}_{2}, \mathrm{WO}_{3}$, and $\mathrm{ZnO}$. Advantages of using MONPs in comparison to metallic NPs are that they are abundant, less expensive, easily modified, biologically compatible, and most importantly bring additional functionalities to the system. For example, $\mathrm{Fe}_{3} \mathrm{O}_{4} \mathrm{NPs}$ have been widely used in magnetically assisted bioseparations, controlled drug delivery, and magnetic resonance imaging, ${ }^{21}$ while $\mathrm{TiO}_{2} \mathrm{NPs}$ are widely used in nanoformulations such as sun creams and are of great

Received: May 16, 2012

Revised: July 4, 2012 
interest for applications in dye-sensitized solar cells, ${ }^{22}$ catalysis, $^{23}$ and biosensors. ${ }^{24}$ Probing enhanced Raman scattering signals by utilizing MONERS therefore opens up significant opportunities in a variety of fields involving metal oxide materials and their interfaces. We demonstrate this by directly monitoring an interfacial chemical reaction and show an exemplar application utilizing the advantages of a MONERS system by tracking the photocatalytic decomposition of an organic dye mediated by $\mathrm{TiO}_{2}$ NPs.

To demonstrate MONERS, we coated a monolayer of 4,4'dimercaptostilbene (dithiol) on flat $\mathrm{Au}$ substrates, and the respective NPs were then allowed to adsorb onto the monolayer, as illustrated schematically in Figure 1a. At the

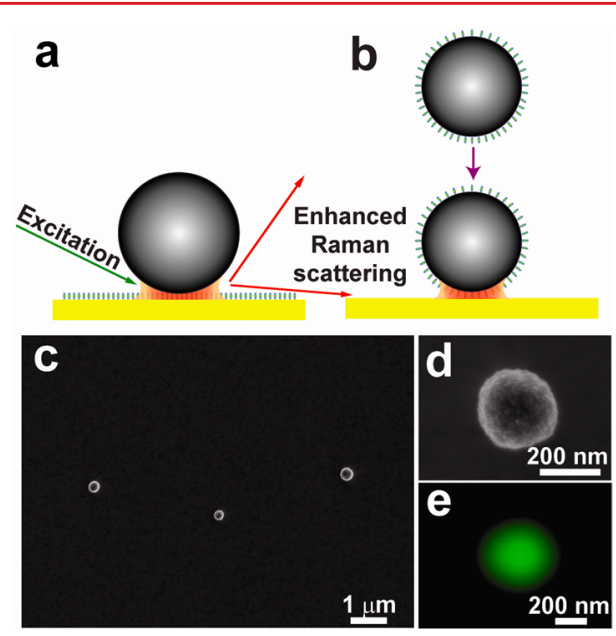

Figure 1. (a) Schematic of a MONP on a functionalized Au surface and (b) schematic of a functionalized MONP brought onto a bare $\mathrm{Au}$ surface. (c) A representative SEM image of well-separated $\mathrm{Fe}_{3} \mathrm{O}_{4} \mathrm{NPs}$. (d) An SEM image of an $\mathrm{Fe}_{3} \mathrm{O}_{4} \mathrm{NP}$ and (e) a MONERS image of an $\mathrm{Fe}_{3} \mathrm{O}_{4} \mathrm{NP}$ on a dithiol-functionalized Au surface obtained by excitation with a $532 \mathrm{~nm}$ laser. The MONERS image is reconstructed by using the integrated intensities of the 1581 and $1628 \mathrm{~cm}^{-1}$ peaks of the dithiol molecule.

outset, we investigated $\mathrm{Fe}_{3} \mathrm{O}_{4} \mathrm{NPs}$ (single $\mathrm{Fe}_{3} \mathrm{O}_{4} \mathrm{NPs}$; average diameter $254 \pm 71 \mathrm{~nm}$ ). The MONERS effect is generated at the single-particle level; to show this, the concentration of suspensions and the adsorption time were controlled such that the $\mathrm{Fe}_{3} \mathrm{O}_{4}$ NPs were well separated from each other (Figure 1c). Figures $1 \mathrm{~d}$ and 1e show respectively an SEM image and a MONERS image for a typical $\mathrm{Fe}_{3} \mathrm{O}_{4} \mathrm{NP}$ on a dithiolfunctionalized $\mathrm{Au}$ surface. Intense molecular signals are observed localized to the $\mathrm{Fe}_{3} \mathrm{O}_{4} \mathrm{NP}$ spot only (Figure 1e). Without the attachment of $\mathrm{Fe}_{3} \mathrm{O}_{4} \mathrm{NPs}$, no Raman signal from the dithiol was detected. To rule out the possibility that roughness might play a role in the enhancement, $\mathrm{Au}$ surfaces with a very high degree of flatness were prepared by a templatestripping method against mica. ${ }^{25}$ These $\mathrm{Au}$ surfaces, with a root-mean-square (rms) roughness of $0.3 \mathrm{~nm}$, showed similar levels of enhancement to the normal flat Au surfaces fabricated by thermal evaporation (Supporting Information Figure S1). Neither type of Au substrate showed any molecular signal in the absence of MONPs, confirming that Raman enhancements indeed only arise from the interaction between the MONPs and $\mathrm{Au}$ surfaces. Although we primarily discuss here the case where molecules have been adsorbed on the surfaces before NP attachment, the MONERS effect works equally well with molecules also adsorbed on NPs (Figure 1b and data shown in Figure S2).

To explore the universality of the MONERS effect, we carried out experiments with a variety of MONPs (Table 1) in

Table 1. Average Experimental EFs for Various NPDithiol-Au Systems ${ }^{a}$

\begin{tabular}{lccc}
\multicolumn{1}{c}{$\mathrm{NPs}$} & $\mathrm{EF} \times 10^{3}(532 \mathrm{~nm})$ & $\mathrm{EF} \times 10^{3}(633 \mathrm{~nm})$ & $n(633 \mathrm{~nm})$ \\
$\mathrm{sFe}_{3} \mathrm{O}_{4}(250 \mathrm{~nm})$ & $7.3(6.0)$ & $17 \pm 14$ & $2.35^{31}$ \\
$\mathrm{cFe}_{3} \mathrm{O}_{4}(22 \mathrm{~nm})$ & $3.9 \pm 2.7$ & $9.7 \pm 7.6$ & $2.35^{31}$ \\
$\mathrm{TiO}_{2}$-rutile & $4.2 \pm 3.2$ & $23 \pm 15$ & $2.49^{32}$ \\
$\mathrm{WO}_{3}$ & $7.7 \pm 5.9$ & $4.5 \pm 5.2$ & $2.10^{33}$ \\
$\mathrm{ZnO}$ & $4.3 \pm 5.3$ & $2.5 \pm 3.6$ & $1.99^{34}$ \\
$100 \mathrm{~nm} \mathrm{AuNP}$ & $150 \pm 160$ & $6400 \pm 5200$ &
\end{tabular}

${ }^{a}$ Data were collected from $30-50$ different NP spots. The $8 \mathrm{~b}$ band $\left(1581 \mathrm{~cm}^{-1}\right)$ of the dithiol is used for calculating the EF values. The refractive indices have been taken from the literature. Standard deviation is indicated in brackets.

addition to the single $\mathrm{Fe}_{3} \mathrm{O}_{4}\left(\mathrm{sFe}_{3} \mathrm{O}_{4}\right)$ NPs mentioned above, each possessing a different crystalline structure (Figure S3), band gap, and refractive index (Table S1). MONERS spectra collected from different MONPs with the dithiol molecules on the surface are shown in Figure 2 (obtained with $532 \mathrm{~nm}$ laser

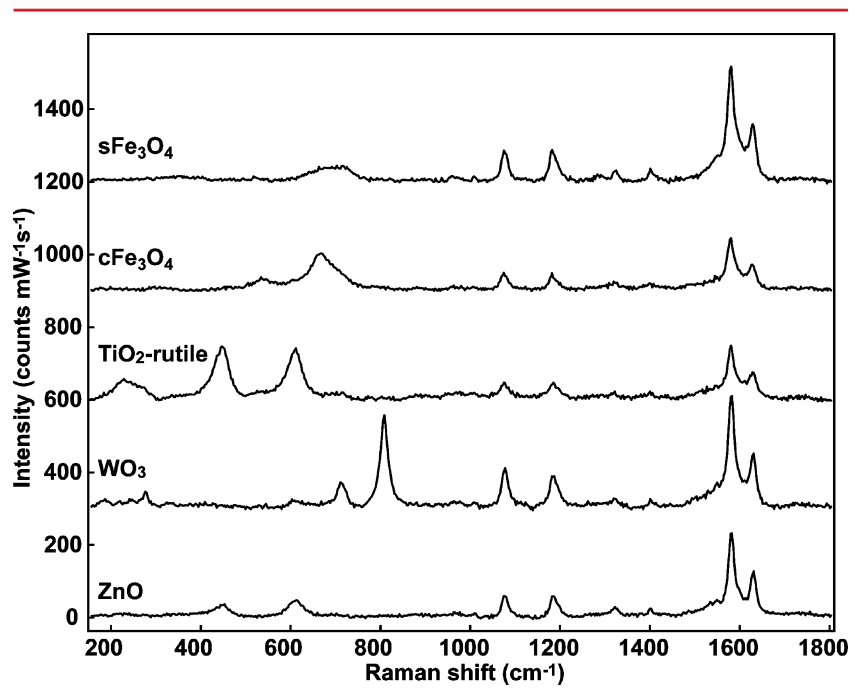

Figure 2. MONERS spectra from single NP $\mathrm{Fe}_{3} \mathrm{O}_{4}\left(\mathrm{sFe}_{3} \mathrm{O}_{4}\right)$ as well from a cluster composed of smaller NPs of $\mathrm{Fe}_{3} \mathrm{O}_{4}\left(\mathrm{cFe}_{3} \mathrm{O}_{4}\right), \mathrm{TiO}_{2^{-}}$ rutile, $\mathrm{WO}_{3}$, and $\mathrm{ZnO}$ on dithiol-functionalized Au surfaces with 532 $\mathrm{nm}$ excitation. The backgrounds have been subtracted, and the spectra are offset for clarity.

excitation) and Figure S4 (obtained with $633 \mathrm{~nm}$ laser excitation), respectively. It is evident that all of these display the MONERS effect. This was again surprising because some of these MONPs were irregularly shaped, and a few of them formed agglomerates (typically 100-400 nm; see Figure S5). In the case of $\mathrm{cFe}_{3} \mathrm{O}_{4}, \mathrm{WO}_{3}$, and $\mathrm{ZnO}$, even though the particles used were clusters composed of small NPs, remarkably large MONERS signals were still observed from molecules on the Au surface. Strong MONERS signals were also obtained with $\mathrm{TiO}_{2}$-rutile NPs as well as $\mathrm{TiO}_{2}$-anatase and the commercially available $\mathrm{TiO}_{2}-\mathrm{P} 25$ (Degussa) NPs (data not shown). In all the MONERS spectra shown in Figure 2, four distinct vibrational modes of dithiol are clearly observed, at $1078 \mathrm{~cm}^{-1}$ (ring mode7a), $1186 \mathrm{~cm}^{-1}$ (ring mode-9a), $1581 \mathrm{~cm}^{-1}$ (ring mode- $8 \mathrm{~b}$ ), 
and $\left.1628 \mathrm{~cm}^{-1}\left(\nu_{(\mathrm{C}=\mathrm{C})}\right)\right)^{26}$ In addition to the vibrational modes of dithiol, some bands are also observed below $900 \mathrm{~cm}^{-1}$. These are characteristic of the MONPs used and are attributed to various phonon modes of the metal oxides, as also confirmed by the bulk Raman spectra collected from the corresponding MONP powders (Figure S6).

The enhancements obtained in the MONERS system were typically 3-4 orders of magnitude compared to corresponding normal Raman measurements (Table 1). These are comparable to or higher than what has been achieved from nanostructured transition metals themselves, ${ }^{27,28}$ for which reported enhancement values range between 10 and $10^{4}$. The reported enhancements obtained for the transition metals, $\mathrm{Fe}$ $(\times 1942)^{29}$ and $\mathrm{Zn}(\times 221),{ }^{30}$ are lower than the enhancements with MONERS. This is due to the higher absorption (interband transitions) in the VIS and NIR regions in transition metals compared to the transition-metal oxides investigated here. Although the enhancements are lower than in an analogous AuNP-on-Au system (Table 1 and Figures S7 and S8), ${ }^{17,18}$ they are sufficiently intense to give high signal-to-noise ratios in MONERS spectra (Figure 2). Signals could be repeatedly recorded from the same NP more than 50 times under conditions below the degradation threshold. Variations in enhancement factors (EFs) across all systems are a manifestation of nanoscale differences in size, shape, and nanoscale geometry. Nevertheless, given the large enhancements, especially compared to transition-metal nanostructures and the multifunctional aspects of metal oxides, MONERS holds enormous potential to study interfacial phenomena.

In order to interpret the observed results in terms of an EM mechanism, finite-element simulations (using the COMSOL Multiphysics software package) were carried out on the $\mathrm{Fe}_{3} \mathrm{O}_{4}$ $\mathrm{NP}$ on $\mathrm{Au}$ system with a gap distance of $1.3 \mathrm{~nm}$, corresponding to the presence of the dithiol molecule. ${ }^{35}$ Because of the weak spectral dependence, we consider enhancements in SERS from the quantity $E^{4}$, where $E$ denotes $\left|E_{y} / E_{0}\right|$. Here, $E_{y}$ refers to the localized scattered field in the $y$-direction midway in the gap between the NP and the Au surface (Figure S9), and $E_{0}$ refers to the field in the absence of the particle. We used three laser sources $\left(\lambda_{\text {ex }}\right)$ in our experiments at 532,633 , and $785 \mathrm{~nm}$. For a $200 \mathrm{~nm} \mathrm{Fe}{ }_{3} \mathrm{O}_{4} \mathrm{NP}$, the simulated $\left|E_{y} / E_{0}\right|^{4}$ is highest for $633 \mathrm{~nm}$ excitation and lowest for $785 \mathrm{~nm}$ excitation (Figure S10). This is indeed consistent with the observed experimental EFs of the $\mathrm{sFe}_{3} \mathrm{O}_{4}$ MONERS system under excitation with different wavelengths (Figure S10). However the broad MONERS resonance is effective over a much wider wavelength range than traditional plasmonic enhancements.

Figure 3a and Figure S11 display the effect of the NP size on the enhancement over the vis-NIR range in the $\mathrm{Fe}_{3} \mathrm{O}_{4} \mathrm{NP}-\mathrm{Au}$ system under a p-polarized field at angles of incidence of $45^{\circ}$ and $0^{\circ}$ to the surface normal, respectively. Clearly, the enhancements increase with increasing NP diameter up to $300 \mathrm{~nm}$ and then show a slight decrease. This is due to an increase in the scattering cross section with size, which, after a critical size, is radiatively damped due to depolarization effects across the particle. ${ }^{36}$ The simulated scattering cross section at a $45^{\circ}$ angle of incidence for $200 \mathrm{~nm} \mathrm{Fe} \mathrm{O}_{4} \mathrm{NPs}$ is overlaid on the measured dark-field scattering spectra in Figure $3 \mathrm{~b}$. There is a good correspondence between them, as the size of the $\mathrm{Fe}_{3} \mathrm{O}_{4}$ NPs used here is of the order of $200 \mathrm{~nm}$. The red-shift observed with increasing $\mathrm{Fe}_{3} \mathrm{O}_{4} \mathrm{NP}$ size is very similar to the well-known size dependence of SPR wavelengths found in all metallic NP systems. $^{37}$
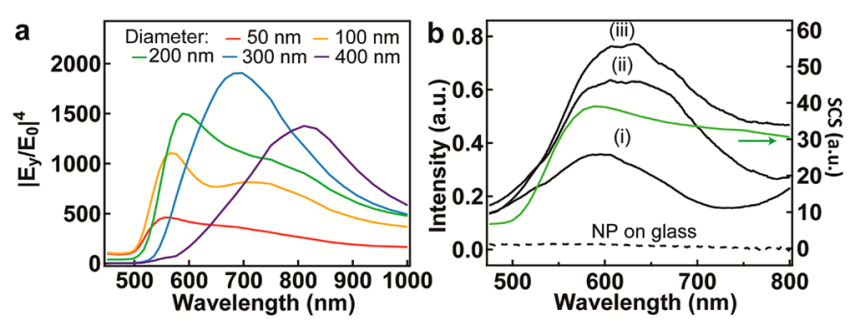

C
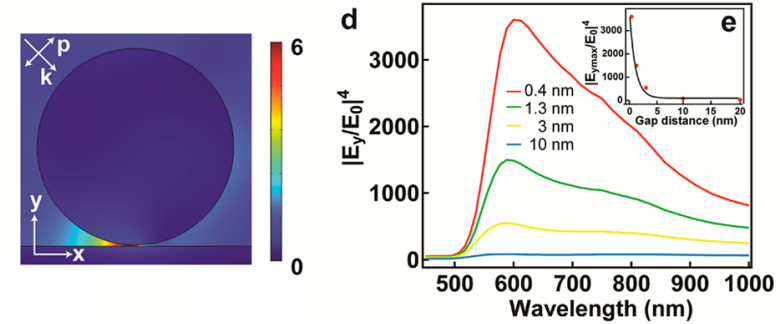

Figure 3. (a) Simulations of the NP size dependence of $\left|E_{y} / E_{0}\right|^{4}$; the gap distance is $1.3 \mathrm{~nm}$. (b) Dark-field scattering spectra (i-iii) of three representative $\mathrm{Fe}_{3} \mathrm{O}_{4} \mathrm{NPs}$ on $\mathrm{Au}$ and on glass overlaid with a simulated spectrum (green). (c) Simulated $\left|E_{y} / E_{0}\right|$ distribution at $633 \mathrm{~nm}$ around a $200 \mathrm{~nm} \mathrm{Fe}{ }_{3} \mathrm{O}_{4} \mathrm{NP}$ on Au with a gap distance of $1.3 \mathrm{~nm}$ with $633 \mathrm{~nm}$ excitation. (d) Simulated spectral variation of $\left|E_{y} / E_{0}\right|^{4}$ for various values of gap distance. (e) $\left|E_{y \max } / E_{0}\right|^{4}$ of a $200 \mathrm{~nm} \mathrm{Fe} \mathrm{O}_{3} \mathrm{NP}$ on $\mathrm{Au}$ as a function of $d / R$, which can be fitted by a power-law function (black curve). ${ }^{38}$ p-polarized light at an incident angle of $45^{\circ}$ was used.

The simulation results indicate that the observed enhancement in MONERS is indeed due to optical modulation from the local dielectric perturbation by the MONPs. When a $\mathrm{Fe}_{3} \mathrm{O}_{4}$ $\mathrm{NP}$ is brought very close to the Au surface, the local dielectric constant is altered due to an EM interaction with the continuum of delocalized propagating surface plasmons at the underlying $\mathrm{Au}$ surface, leading to a localization of the electric field in the gap formed between the NP and the metal surface. An alternative view considers enhanced scattering by the highindex NP exciting surface plasmons on a flat Au surface, which become confined in the gap, producing a large EM-field enhancement. While coupling to flat metal surfaces is impossible due to the momentum mismatch between the wave vectors of a free-space photon and a plasmon confined to a surface, the high-index NP acts as an antenna coupling light into the gap above the metal surface. Although electric dipoles $^{39}$ and array of dielectric spheres ${ }^{40}$ near a metallic surface as well the case of a metal NP on a high dielectric substrate $^{19}$ have been predicted to enhance scattering, the coupling and enhancement with a high refractive index MONP on a plasmonic surface are uniquely examined here.

The evidence that indeed excitation of localized surface plasmons of the substrate occurs on bringing MONPs close to the surface is seen in experiments with Pt which is only weakly plasmonic. Only a weak MONERS signal can be detected (data not shown) although thiols form well-organized self-assembled monolayers on Pt similar to Au. ${ }^{41}$ Clearly the size and type of NP material are important in MONERS, but the plasmonic properties of the substrate are significant. A dielectric surface does not give any enhancement; $\mathrm{Fe}_{3} \mathrm{O}_{4} \mathrm{NPs}$ on glass gave no enhancement and yield an MONERS EF of only 23 in simulations (Figure S12). Furthermore, by comparing the same vibrational modes of different molecules between Au NP on an $\mathrm{Au}$ surface and $\mathrm{sFe}_{3} \mathrm{O}_{4}$-based MONERS systems, we find consistent shifts in vibrational frequencies across all bands due to different chemical interactions but little difference in 
enhancements for the different peaks (Figure S13). Also, the molecules mixed with MONPs alone do not produce any enhanced Raman signals (Figure S14). Therefore, we rule out a "chemical effect" as a mechanism for the observed enhancements in MONERS.

The highest field enhancement is localized in the gap between the $\mathrm{Fe}_{3} \mathrm{O}_{4} \mathrm{NP}$ and the $\mathrm{Au}$ substrate (Figure 3c and Figure S9). As expected, the enhancement effect in MONERS is highly sensitive to the size of the gap (Figures 3d,e). The distribution of $E^{4}$ becomes tighter and rapidly increases as the gap distance is decreased, due to higher field confinement and scales as $(d / R)^{-1 / 2}$. Indeed, higher MONERS enhancements of $(1.6 \pm 1.7) \times 10^{5}$ were obtained with 4-mercaptobenzoic acid (MBA), where the MBA molecule is approximately half the length of the dithiol molecule. The intense enhancement obtained from MBA, along with many other dyes and molecules (Figure S15), also proves that the enhancements demonstrated here show no selectivity toward probe molecules, unlike the charge-transfer enhancement observed for semiconductor NPs by other researchers. ${ }^{12-14}$

Significantly, no MONERS signals were obtained with dielectric particles, such as $\mathrm{SiO}_{2}$ and polystyrene, under our experimental conditions. Simulations reveal that such $\mathrm{SiO}_{2} \mathrm{NP}-$ based MONERS system should indeed give 2 orders of magnitude lower enhancements (Figure S16). This difference between $\mathrm{Fe}_{3} \mathrm{O}_{4} \mathrm{NP}$ and $\mathrm{SiO}_{2} \mathrm{NP}$ systems indicates that the field enhancement is influenced by the refractive index of the NP. The correlation between refractive indices and EFs at $633 \mathrm{~nm}$ is evident since the observed MONERS EFs of $\mathrm{ZnO}$ and $\mathrm{WO}_{3}$ are indeed lower than those of $\mathrm{TiO}_{2}$-rutile and $\mathrm{Fe}_{3} \mathrm{O}_{4}$ (Table 1). This is consistent with our explanation that a higher refractive index NP leads to more efficient scattering with better confinement of the scattered field in the gap.

A key property of $\mathrm{Fe}_{3} \mathrm{O}_{4} \mathrm{NPs}$ is that they are ferromagnetic and hence, are used in bioseparations. They assemble into linear chains under the influence of an external magnetic field (Figure 4a). These chains are formed by single layers of $\mathrm{Fe}_{3} \mathrm{O}_{4}$ NPs. As clearly seen from Raman map images (Figure 4b), the intense signals are localized only to the assembled chains of NPs. Spectra obtained from MONERS chains under excitation from both 532 and $633 \mathrm{~nm}$ pump light (Figures 4c,d) show signals from molecules under the chains. This confirms the field enhancement is maximized in the gap between the particles and the surface, rather than between the particles themselves. This is further confirmed in simulations of a dimer on the surface (Figure 4e and Figure S17), which shows that field enhancements between the particles are very small compared to those in the gap between the particle and the substrate. In this case there is a very weak interaction between the MONPs themselves unlike metallic NPs which are plasmon active. Thus, the ferromagnetic property in tandem with the MONERS approach could be used to separate and detect biomolecules in situ in solutions in real time.

Furthermore, MONPs can not only assist in monitoring the molecular transformation at an interface but also actively catalyze it. We monitored the photocatalytic decomposition of methylene blue (MB) by $\mathrm{TiO}_{2}$ NPs (P25, Degussa) by MONERS (Figure 5). The spectra in Figure 5 b clearly show that with increased exposure to UV radiation the characteristic Raman peaks of MB decrease in intensity. However, comparing the different Raman modes in the spectra the molecular mechanism can be elucidated. The 448, 500, 1510, and 1624 $\mathrm{cm}^{-1}$ peaks are assigned to $\mathrm{C}-\mathrm{N}-\mathrm{C}, \mathrm{C}-\mathrm{S}-\mathrm{C},-\mathrm{NH}_{2}$
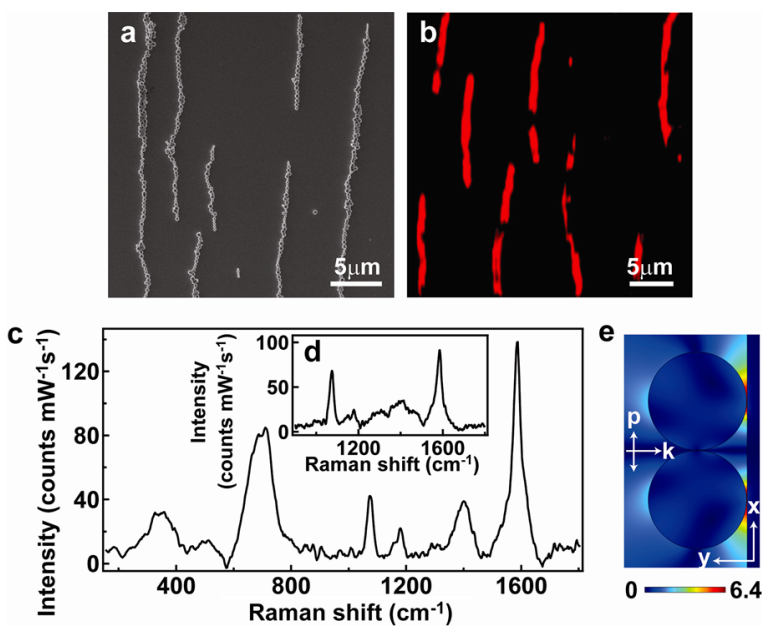

Figure 4. (a) An SEM image and (b) a MONERS image collected under excitation with a $633 \mathrm{~nm}$ laser of representative $\mathrm{Fe}_{3} \mathrm{O}_{4}$ lines assembled on an MBA-functionalized Au surface under the influence of an external magnetic field. The MONERS image is reconstructed by using the integrated intensities of the $1588 \mathrm{~cm}^{-1}$ peak of MBA. MONERS spectra from a spot on an $\mathrm{Fe}_{3} \mathrm{O}_{4}$ line on an MBAfunctionalized Au surface under excitations with (c) $532 \mathrm{~nm}$ and (d) $633 \mathrm{~nm}$ lasers. The backgrounds have been subtracted. (e) Simulated $\left|E_{y} / E_{0}\right|$ distribution at $633 \mathrm{~nm}$ around a $200 \mathrm{~nm}$ diameter $\mathrm{Fe}_{3} \mathrm{O}_{4} \mathrm{NP}$ dimer on $\mathrm{Au}$ under excitation with $633 \mathrm{~nm}$ at an incident angle of $0^{\circ}$. The gap distances between $\mathrm{Fe}_{3} \mathrm{O}_{4} \mathrm{NPs}$ and between dimer and substrate are both $1.3 \mathrm{~nm} . E_{y}$ is the local field in the $y$ direction; $E_{0}$ is the field in absence of the NP.

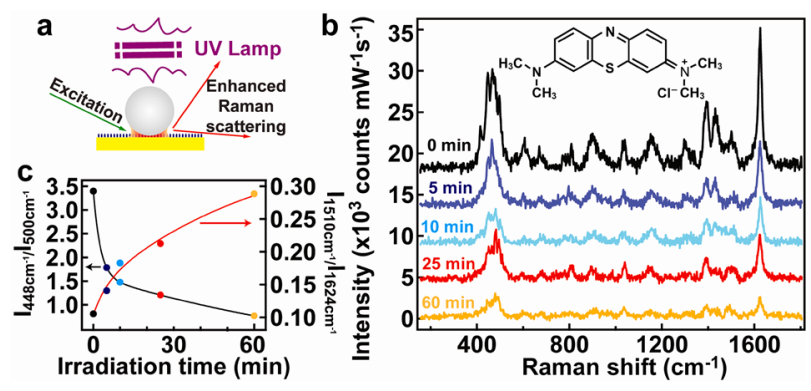

Figure 5. (a) Schematic of MONP mediated photocatalysis and simultaneous MONERS monitoring. (b) MB spectra after different exposures to UV irradiation showing rapid decrease of the peaks. MB molecular structure is shown in inset. (c) Ratio of intensities of the 448 to $500 \mathrm{~cm}^{-1}$ peaks decreases, indicating predominant cleavage of $\mathrm{C}-\mathrm{N}-\mathrm{C}$ bond, while that of the 1510 to $1624 \mathrm{~cm}^{-1}$ peaks increases as a result of ring scission, leading to formation of $-\mathrm{NH}_{2}$.

deformation, and $\mathrm{C}-\mathrm{C}$ ring stretching modes, respectively. ${ }^{42}$ Figure $5 \mathrm{c}$ shows that the $\mathrm{C}-\mathrm{N}-\mathrm{C}$ bond is cleaved in preference to the $\mathrm{C}-\mathrm{S}-\mathrm{C}$ bond leading to a decrease in the ratio of the corresponding peaks. The simultaneous increase in the peak ratio of the $-\mathrm{NH}_{2}$ deformation to the benzene ring mode agrees with the above and confirms predominant scission of the central ring vis-a-vis the adjacent benzene moieties, providing direct evidence of the degradation mechanism proposed for MB on $\mathrm{TiO}_{2}{ }^{43}$ This clearly demonstrates that MONERS allows direct molecular observation and understanding of chemical processes at a metal oxide interface.

In conclusion, the straightforward origin of the MONERS effect demonstrated here gives enhanced Raman scattering mediated by MONPs which has never been previously attempted. Although the construction is analogous to the 
metallic ( $\mathrm{Au}$ or $\mathrm{Ag}$ ) NP-on-substrate systems $^{17-20}$ it is fundamentally different since MONPs are nonplasmonic and the mechanism of coupling light into metallic surfaces is through optical effects induced by the high-index particle. This system can be similarly extended to other NPs with high refractive index, such as silicon and carbon NPs; further experiments are currently in progress. More importantly, this work opens up a significant area for the fundamental and applied study of chemically active surfaces not possible with the all metallic systems. We have shown initial proof-of-concept experiments to exemplify these distinct advantages, using MONPs to directly monitor and elucidate the mechanism of photocatalytic decomposition of MB. MONERS will thus aid understanding many types of interfacial phenomena, including those in catalysis and energy transfer in photovoltaics and photoelectrochemical systems, besides adding novel functionalities to traditional methods of detection and biosensing.

\section{ASSOCIATED CONTENT}

\section{S Supporting Information}

Experimental details; Figures S1-S17. This material is available free of charge via the Internet at http://pubs.acs.org.

\section{AUTHOR INFORMATION}

\section{Corresponding Author}

*E-mail:sm735@cam.ac.uk.

\section{Notes}

The authors declare no competing financial interest.

\section{ACKNOWLEDGMENTS}

We acknowledge funding from EPSRC (EP/H028757/1, EP/ H007024/1, EP/G060649/1, and EU CUBIHOLES) for this work. We also thank Nokia Research Centre, Cambridge, for studentship support (L.L.) and Trinity College Cambridge for a scholarship (T.H.).

\section{REFERENCES}

(1) Hwang, H. Y.; Iwasa, Y.; Kawasaki, M.; Keimer, B.; Nagaosa, N.; Tokura, Y. Nat. Mater. 2012, 11, 103.

(2) Rhodes, C.; Franzen, S.; Maria, J.-P.; Losego, M.; Leonard, D. N.; Laughlin, B.; Duscher, G.; Weibel, S. J. Appl. Phys. 2006, 100, 054905.

(3) Li, S. Q.; Guo, P.; Zhang, L.; Zhou, W.; Odom, T. W.; Seideman, T.; Ketterson, J. B.; Chang, R. P. H. ACS Nano 2011, 5, 9161.

(4) Kanehara, M.; Koike, H.; Yoshinaga, T.; Teranishi, T. J. Am. Chem. Soc. 2009, 131, 17736.

(5) Losego, M. D.; Efremenko, A. Y.; Rhodes, C. L.; Cerruti, M. G.; Franzen, S.; Maria, J.-P. J. Appl. Phys. 2009, 106, 024903.

(6) Dominici, L.; Michelotti, F.; Brown, T. M.; Reale, A.; Di Carlo, A. Opt. Express 2009, 17, 10155.

(7) Nie, S.; Emory, S. R. Science 1997, 275, 1102.

(8) Cao, Y. C.; Jin, R.; Mirkin, C. A. Science 2002, 297, 1536.

(9) Kneipp, K.; Wang, Y.; Kneipp, H.; Perelman, L. T.; Itzkan, I.; Dasari, R. R.; Feld, M. S. Phys. Rev. Lett. 1997, 78, 1667.

(10) Moskovits, M. Rev. Mod. Phys. 1985, 57, 783.

(11) Otto, A. J. Raman Spectrosc. 2005, 36, 497.

(12) Musumeci, A.; Gosztola, D.; Schiller, T.; Dimitrijevic, N. M.; Mujica, V.; Martin, D.; Rajh, T. J. Am. Chem. Soc. 2009, 131, 6040.

(13) Tarakeshwar, P.; Finkelstein-Shapiro, D.; Hurst, S. J.; Rajh, T.; Mujica, V. J. Phys. Chem. C 2011, 115, 8994.

(14) Wang, X.; Shi, W.; She, G.; Mu, L. Phys. Chem. Chem. Phys. 2012, 14, 5891.

(15) Li, J. F.; Huang, Y. F.; Ding, Y.; Yang, Z. L.; Li, S. B.; Zhou, X. S.; Fan, F. R.; Zhang, W.; Zhou, Z. Y.; Wu, D. Y.; Ren, B.; Wang, Z. L.; Tian, Z. Q. Nature 2010, 464, 392.
(16) Li, J.-F.; Ding, S.-Y.; Yang, Z.-L.; Bai, M.-L.; Anema, J. R.; Wang, X.; Wang, A.; Wu, D.-Y.; Ren, B.; Hou, S.-M.; Wandlowski, T.; Tian, Z.-Q. J. Am. Chem. Soc. 2011, 133, 15922.

(17) Rycenga, M.; Xia, X.; Moran, C. H.; Zhou, F.; Qin, D.; Li, Z.-Y.; Xia, Y. Angew. Chem., Int. Ed. 2011, 50, 5473.

(18) Hill, R. T.; Mock, J. J.; Urzhumov, Y.; Sebba, D. S.; Oldenburg, S. J.; Chen, S.-Y.; Lazarides, A. A.; Chilkoti, A.; Smith, D. R. Nano Lett. 2010, 10, 4150.

(19) Knight, M. W.; Wu, Y.; Lassiter, J. B.; Nordlander, P.; Halas, N. J. Nano Lett. 2009, 9, 2188.

(20) Mubeen, S.; Zhang, S.; Kim, N.; Lee, S.; Krämer, S.; Xu, H.; Moskovits, M. Nano Lett. 2012, 12, 2088.

(21) Mornet, S.; Vasseur, S.; Grasset, F.; Duguet, E. J. Mater. Chem. 2004, 14, 2161.

(22) Guldin, S.; Hüttner, S.; Kolle, M.; Welland, M. E.; MüllerBuschbaum, P.; Friend, R. H.; Steiner, U.; Tétreault, N. Nano Lett. 2010, 10, 2303.

(23) Hoffmann, M. R.; Martin, S. T.; Choi, W.; Bahnemann, D. W. Chem. Rev. 1995, 95, 69.

(24) Zhou, H.; Gan, X.; Wang, J.; Zhu, X.; Li, G. Anal. Chem. 2005, 77,6102 .

(25) Hugall, J. T.; Finnemore, A. S.; Baumberg, J. J.; Steiner, U.; Mahajan, S. Langmuir 2012, 28, 1347.

(26) Osawa, M.; Matsuda, N.; Yoshii, K.; Uchida, I. J. Phys. Chem. 1994, 98, 12702.

(27) Tian, Z.-Q.; Ren, B.; Wu, D.-Y. J. Phys. Chem. B 2002, 106, 9463.

(28) Abdelsalam, M. E.; Mahajan, S.; Bartlett, P. N.; Baumberg, J. J.; Russell, A. E. J. Am. Chem. Soc. 2007, 129, 7399.

(29) Cao, P. G.; Yao, J. L.; Ren, B.; Mao, B. W.; Gu, R. A.; Tian, Z. Q. Chem. Phys. Lett. 2000, 316, 1.

(30) Gu, R.-A.; Shen, X.-Y.; Liu, G.-K.; Ren, B.; Tian, Z.-Q. J. Phys. Chem. B 2004, 108, 17519.

(31) Schlegel, A.; Alvarado, S. F.; Wachter, P. J. Phys. C: Solid State Phys. 1979, 12, 1157.

(32) Bass, M. Handbook of Optics, 3rd ed.; McGraw-Hill: New York, 2009; Vol. IV.

(33) Rao, M. C.; Hussain, O. M. Res. J. Chem. Sci. 2011, 1, 76.

(34) Bass, M. Handbook of Optics, 2nd ed.; McGraw-Hill: New York, 1994; Vol. II.

(35) Guarrotxena, N.; Ren, Y.; Mikhailovsky, A. Langmuir 2011, 27, 347.

(36) Blaber, M. G.; Schatz, G. C. Chem. Commun. 2011, 11, 1221.

(37) Driskell, J. D.; Lipert, R. J.; Porter, M. D. J. Phys. Chem. B 2006, $110,17444$.

(38) Huang, F.; Baumberg, J. J. Nano Lett. 2010, 10, 1787.

(39) Xiao, M.; Zayats, A.; Siqueiros, J. Phys. Rev. B 1997, 55, 1824.

(40) Inoue, M. Pure Appl. Chem. 1987, 59, 1253.

(41) Love, J. C.; Estroff, L. A.; Kriebel, J. K.; Nuzzo, R. G.; Whitesides, G. M. Chem. Rev. 2005, 105, 1103.

(42) Hutchinson, K.; Hester, R. E.; Albery, W. J.; Hillman, A. R. J. Chem. Soc., Faraday Trans. 1 1984, 80, 2053.

(43) Houas, A.; Lachheb, H.; Ksibi, M.; Elaloui, E.; Guillard, C.; Herrmann, J.-M. Appl. Catal. B: Environ. 2001, 31, 145. 